\title{
Moments of the quark electromagnetic-current two-point function at the physical point: connected contributions
}

\author{
Budapest-Marseille-Wuppertal Collaboration \\ K. Miura $^{1}{ }^{*}$, Sz. Borsanyi ${ }^{2}$, Z. Fodor ${ }^{2,3,4}$, T. Kawanai ${ }^{4}$, S. Krieg ${ }^{2,4}$, L. Lellouch ${ }^{1}$, \\ R. Malak ${ }^{1,5}$, K.K. Szabo ${ }^{2,4}$, C. Torrero ${ }^{1}$, and B.C. Toth ${ }^{2}$ \\ ${ }^{1}$ Aix-Marseille U., U. de Toulon, CNRS, CPT, UMR 7332, F-13288 Marseille, France \\ ${ }^{2}$ Department of Physics, Bergische Universität Wuppertal, Gaussstr. 20, D-42119 Wuppertal, \\ Germany \\ ${ }^{3}$ Inst. for Theor. Physics, Eötvös University, Pázmány P. sét. 1/A, H-1117 Budapest, Hungary \\ ${ }^{4}$ Jülich Supercomputing Centre, Forschungszentrum Jülich, D-52425 Jülich, Germany \\ ${ }^{5}$ CNRS, CEA, Maison de la Simulation, USR 3441, F-91191 Gif-sur-Yvette Cedex, France
}

E-mail: kohtaroh.miura@cpt.univ-mrs.fr

\begin{abstract}
The low, euclidean momentum behavior of the hadron vacuum polarization (HVP) is critical for determining, amongst other quantities, the anomalous magnetic moments of the muon. Here we present lattice QCD results for the first two derivatives of the HVP function at vanishing virtuality [1]. Computations are performed with $2+1+1$ flavors of staggered quarks around the physical mass point, in volumes of linear extent larger than $6 \mathrm{fm}$, and at six values of the lattice spacing, allowing for a fully controlled continuum extrapolation. We further consider possible uncertainties which stem from finite-volume and isospin-breaking effects. After adding to our connected contributions the disconnected terms presented in [2], we compare the resulting derivatives of the full HVP with phenomenological estimates.
\end{abstract}

34th annual International Symposium on Lattice Field Theory

24-30 July 2016

University of Southampton, UK

\footnotetext{
* Speaker.
} 


\section{Introduction}

The muon's anomalous magnetic moments $a_{\mu}=\left(g_{\mu}-2\right) / 2$, with $g_{\mu}$ the Landé g-factor, is determined experimentally with a high precision of $0.5 \mathrm{ppm}$ [3], and forthcoming experiments aim to improve uncertainties to around $0.15 \mathrm{ppm}$ [4]. The comparison of these results with Standard Model (SM) predictions, accurate to about $0.4 \mathrm{ppm}$ [5], provides a probe of physics beyond the SM. At present, there is a 3.6 standard-deviation tension between the SM and experiment [6].

The largest source of the uncertainties for $a_{\mu}$ in SM comes from the hadron vacuum polarization (HVP) contribution due to its nonperturbative nature. Today, the HVP is best determined via dispersion relations combined with the cross section data of $e^{+} e^{-}$to hadrons or the rate data of hadronic $\tau$ decays [7]. However, since the pioneering work of [8], significant progresses have been made in the lattice QCD calculations of the leading order (LO) HVP contributions $\left(a_{\mu}^{\mathrm{LO}-\mathrm{HVP}}\right)$ to $a_{\mu}$. Moreover, in the long run, this approach is likely to represent the most cost-effective way to increase the precision of the HVP to the levels that will soon be required by the new round of measurements of $a_{\mu}$ [4] and, more generally, by particle physics phenomenology.

The low, euclidean momentum behavior of the HVP, determined in great part by its slope and curvatures at vanishing virtuality, is critical for computing $a_{\mu}^{\mathrm{LO}-\mathrm{HVP}}$. In this contribution, we present a full lattice QCD calculation of the first two derivatives of the HVP in the isospin limit, including the contributions from $u, d, s$, and $c$ quarks in the vicinity of the physical mass point. The derivatives are computed, by Fourier transform, from spacetime moments of the quark electromagnetic current two-point function. Here we focus on the quark-connected contributions. The quarkdisconnected contributions are discussed separately in Ref. [2]. We discuss possible uncertainties which stem from finite-volume and isospin-breaking effects. We add to the results presented here the disconnected contributions discussed in [2] and compare the total moments obtained with phenomenological estimates.

\section{Simulations}

We employ the tree-level improved Symanzik gauge action [9] and a fermion action for four flavors of stout-smeared [10] staggered quarks. The up and down quarks masses are taken to be degenerate $\left(m_{u, d}=m_{l}\right)$, and their ratio to the strange quark mass $\left(m_{s}\right)$ are tuned so that NambuGoldstone (NG) pion and kaon masses are close to their physical values. The charm-quark mass $\left(m_{c}\right)$ is fixed by $m_{c} / m_{s}=11.85$ [11]. To define the physical mass point, we use the isospin corrected pion/kaon masses, $M_{\pi}=134.8 \mathrm{MeV}$ and $M_{K}=494.2 \mathrm{MeV}$ [12]. The lattice spacing (a) from the pion decay constant, using the Wilson-flow-based [13] $w_{0}$-scale [14] at intermediate steps. The trajectories are obtained using the Rational Hybrid Monte Carlo (RHMC) algorithm. The topological charge undergoes sufficient number of tunnelings even on the finest lattices.

As summarized in Table 1, our lattice simulations are carried out in the vicinity of the physical mass point on lattices of spatial extent $L \gtrsim 6 \mathrm{fm}$ and of time extent $T \simeq 9 \div 11 \mathrm{fm}$. The vectorvector current correlator with light quarks is notoriously noisy at large distance. We address this problem by performing a high-precision lattice calculation with 768 random source measurements.

We compute the electromagnetic vector current correlator, $\left\langle j_{\mu}(x) j_{\mu}(0)\right\rangle$, where $j_{\mu} / e=\frac{2}{3} \bar{u} \gamma_{\mu} u-$ $\frac{1}{3} \bar{d} \gamma_{\mu} d-\frac{1}{3} \bar{s} \gamma_{\mu} s+\frac{2}{3} \bar{c} \gamma_{\mu} c$. We use the conserved lattice current at the source and sink so that no renormalization is required. The Fourier transform of $\left\langle j_{\mu}(x) j_{\mu}(0)\right\rangle$ gives the HVP tensor 


\begin{tabular}{cllllll}
\hline \hline$a[\mathrm{fm}]$ & $T$ & $L$ & \#traj. & $M_{\pi}[\mathrm{MeV}]$ & $M_{K}[\mathrm{MeV}]$ & \#SRC (ud,s,c) \\
\hline 0.134 & 64 & 48 & 10000 & $\sim 131$ & $\sim 479$ & $(768,128,64)$ \\
0.118 & 96 & 56 & 15000 & $\sim 132$ & $\sim 483$ & $(768,64,64)$ \\
0.111 & 84 & 56 & 15000 & $\sim 133$ & $\sim 483$ & $(768,64,64)$ \\
0.095 & 96 & 64 & 25000 & $\sim 133$ & $\sim 488$ & $(768,64,64)$ \\
0.078 & 128 & 80 & 35000 & $\sim 133$ & $\sim 488$ & $(768,64,64)$ \\
0.064 & 144 & 96 & 4500 & $\sim 133$ & $\sim 490$ & $(768,64,64)$ \\
\hline \hline
\end{tabular}

Table 1: Details of the simulations and statistics used here.

$\Pi_{\mu v}\left(Q^{2}\right)=\left(\delta_{\mu \nu} \hat{Q}^{2}-\hat{Q}_{\mu} \hat{Q}_{v}\right) \Pi\left(Q^{2}\right)+\cdots$, where $\hat{Q}=(2 / a) \sin \left(n \pi a Q_{\mu} / L_{\mu}\right)$ is the lattice momentum and the "..." denotes lattice artefacts. The scalar HVP $\Pi\left(Q^{2}\right)$ above is then responsible for the non-perturbative hadronic effects in the leading-order (LO, $\mathscr{O}\left(\alpha^{2}\right), \alpha=e^{2} /(4 \pi)$ ) contribution to the muon anomalous magnetic moment: $a_{\mu}^{\mathrm{LO}-\mathrm{HVP}}=(\alpha / \pi)^{2} \int_{0}^{\infty} d Q^{2} \omega\left(m_{\mu}^{2}, Q^{2}\right) \hat{\Pi}\left(Q^{2}\right)$ with $\hat{\Pi}\left(Q^{2}\right)=\Pi\left(Q^{2}\right)-\Pi(0)$. Here, the kernel $\omega\left(m_{\mu}^{2}, Q^{2}\right)$ is a known kinematic function that diverges at $Q^{2}=0$ in such a way that the integrand peaks around the low $Q^{2}$ value of $\left(m_{\mu} / 2\right)^{2}$. Because of this, the first few coefficients of the Taylor expansion of $\hat{\Pi}\left(Q^{2}\right)$ around $Q^{2}=0$ are sufficient to determine $a_{\mu}^{\mathrm{LO}-\mathrm{HVP}}$ accurately $[15,16]$. We obtain these coefficients by computing moments of the correlator $\left\langle j_{\mu}(x) j_{v}(0)\right\rangle$ :

$$
\Pi_{n, \mu v}=(-)^{n+1} \sum_{x}\left(\hat{x}_{v}^{2(n+1)} /(2 n+2) !\right) \operatorname{Re}\left\langle j_{\mu}(x) j_{\mu}(0)\right\rangle, \quad \hat{x}_{v}=\min \left\{x_{v}, L_{v}-x_{v}\right\},
$$

where the $L_{v}=L$ or $T$ is the size of the lattice in the $v$-direction. On an $L^{3} \times T, L<T$, lattice, $\mathrm{O}(4)$ symmetry is reduced to the cubic group. This implies that the moments obtained from different $\mu \nu$ components correspond to different invariants which exponentially converge onto a unique coefficient in the infinite-volume limit. Thus, we consider three different averages which are invariant under spatial cubic transformations: $\Pi_{n, s s}=\sum_{i \neq j} \Pi_{n, i j} / 6, \Pi_{n, t s}=\sum_{j=1,2,3} \Pi_{n, 4 j} / 3$, and $\Pi_{n, s t}=\sum_{i=1,2,3} \Pi_{n, i 4} / 3$. The final results are given by $\Pi_{n}=\left(\Pi_{n, s s}+\Pi_{n, t s}+\Pi_{n, s t}\right) / 3$, while the difference among the three quantities contain information about finite-volume effects. The averaged moment $\Pi_{n}$ is composed of the connected and disconnected contributions, where the former is further decomposed into the light (up and down), strange, and charm contributions: $\Pi_{n}=\Pi_{n}^{\text {con }}+(1 / 9) \Pi_{n}^{\text {disc }}$ with $\Pi_{n}^{\text {con }}=(5 / 9) \Pi_{n}^{l}+(1 / 9) \Pi_{n}^{s}+(4 / 9) \Pi_{n}^{c}$. Here we focus on the connected part $\Pi_{n}^{\text {con }}$, while the disconnected part $\Pi_{n}^{\text {disc }}$ is detailed in another contribution of ours [2].

Let us consider the light component contribution in the temporal component $\Pi_{n, s t}$. We denote it as $\prod_{n, s t}^{l}$, which is characterized by the correlator $C^{l}(t)=\sum_{\mathrm{x}} \sum_{i=1,2,3} \operatorname{Re}\left\langle j_{i}(t, \mathrm{x}) j_{i}(0)\right\rangle /\left.3\right|_{u d}$ (c.f. Eq. (2.1)). The correlator $C^{l}(t)$ can extend up to $T / 2 \lesssim 6 \mathrm{fm}$, and the signal deteriorates quickly with increasing distance. To address this problem, we introduce a cut $t_{c} \mathrm{fm}$ in time, and replace the $C^{l}(t)$ by an upper bound $\left(C^{l}\left(t_{c}\right) \varphi(t) / \varphi\left(t_{c}\right)\right)$ and a lower bound (zero) for $t>t_{c}$. Here, $\varphi(t)=$ $\cosh \left[E_{2 \pi}(T / 2-t)\right]$ with $E_{2 \pi}$ denoting energy of two pions, each with the smallest nonvanishing lattice momentum, for which we use $2 \pi / L$. Typically the $\Pi_{n, s t}^{l}$ obtained with two bounds agree for $t_{c} \gtrsim 3 \mathrm{fm}$. We adopt $t_{c}=3.1 \mathrm{fm}$ and average the $\Pi_{n, s t}^{l}$ obtained with two bounds to get the final result. We confirmed that the corrections to the upper bound from pion-pion interactions are negligible, using the model of Ref. [17] and neglecting four-pion contributions. 


\section{Results}

We fit the lattice results as a function of the lattice spacing squared $a^{2}$ and the pion/kaon masssquared deviations, $\Delta M_{\pi, K}^{2}$, from their physical values. For the $a^{2}$ dependence, we have confirmed that the linear ansatz is enough and adopted in the following. In Fig. 1, we show these fits for the connected part of the first two Taylor coefficients $\Pi_{n}^{l, s, c}, n=1,2$. The final central value and systematic error of the continuum results are obtained in an extended frequentist approach [18].They are, respectively, the mean and standard error of the Akaike-Information-Criterion-weighted distributions obtained by imposing four different cuts on lattice spacing (no cut (fit0), $a \leq 0.118$ (fit1), 0.111 (fit2), 0.095 (fit3) fm), corresponding to the four fit lines in each plot. Owing to the large number of lattice spacings (6) and more generally simulation points (15), we are able to take the continuum limit and interpolate to the physical mass point with full control over systematics error.

In the leftmost panels of Fig. 1, we show the continuum extrapolation of the light component contributions: $\Pi_{n=1}^{l}$ (top) and $\Pi_{n=2}^{l}$ (bottom). The red squares represent our lattice results for each simulation, and they are consistent with the HPQCD results [19] (green triangles). Reasonable fit qualities are achieved without pion/kaon mass correction terms. The coarsest lattice gives a value about $15 \%$ smaller than the continuum limit.

In contrast to the light components, the kaon mass correction term is necessary to obtain a reasonable fit quality for the strange contributions to the moments. In the middle panels of Fig. 1, the kaon-mass corrected results ( $\Pi_{n=1}^{s}$ (top) and $\Pi_{n=2}^{s}$ (bottom)) are shown as red squares, and are compared to the uncorrected results (gray squares) as well as to the HPQCD results [16] (green triangles). Good fit qualities are achieved. The strange channel has much smaller lattice artefacts than the light $(\sim 2 \%)$, since it is much less affected by taste violations.

For the charm contribution, the fit qualities are much worse, because the precision of the data is orders of magnitude better than for the lighter flavors. Here we fit only a random subset of 10 configurations, which increases the statistical error and leads to good fit qualities. Here, mass corrections are needed. In the right panels of Fig. 1, the mass corrected data ( $\Pi_{n=1}^{c}$ (top) and $\Pi_{n=2}^{c}$ (bottom)) are shown as red squares, and are compared to the uncorrected results (gray squares) as well as to the HPQCD results [11]. Results on the coarsest lattice deviate by about $20 \%$ from the continuum limit.

\section{Summary and discussion}

The continuum limit values of the connected contributions to the slope and curvature of the HVP function at vanishing virtuality are summarized in Table 2, together with their disconnected parts [2]. The total lattice uncertainty on $\Pi_{1}$ is $1.8 \%$ and on $\Pi_{2}, 4.4 \%$. However, additional errors may result from finite-volume (FV) and isospin-breaking effects. Here we discuss those corrections and compare our results with phenomenology.

In the absence of a systematic study with simulations in a variety of volumes, only model estimates of FV effects can be made. For large volumes, FV corrections will be governed by pion contributions that can be computed in chiral perturbation theory $(\chi \mathrm{PT})$ [20]. These are largest in the $I=1$ channel which is dominated by two-pion exchange at large distances. For all three index combinations, ss, $t s$ and $s t$ (see text after Eq. (2.1)), we compute the FV corrections for $\Pi_{n}^{l}$ at one loop in $\chi$ PT. These are shown as a function of box size $L$ in Fig. 2, for $n=1,2$ and $T / L=3 / 2$. We 

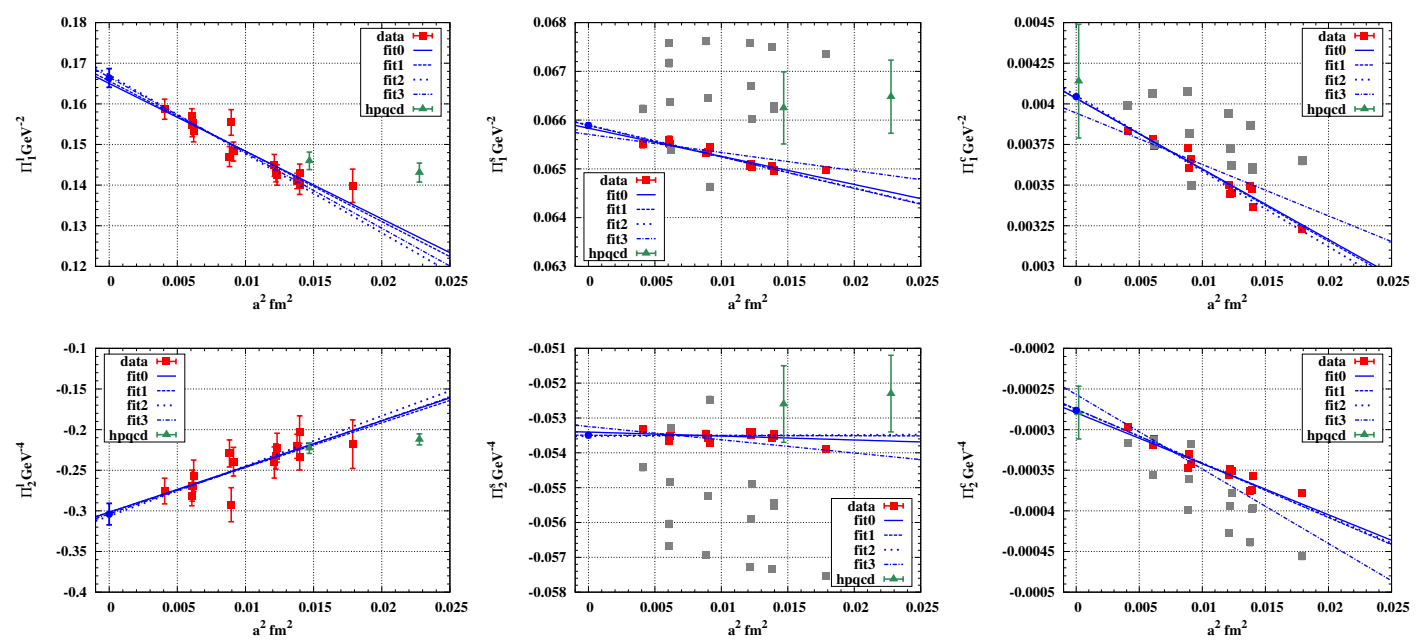

Figure 1: Continuum extrapolations of the light (left), strange (middle), and charm (right) contributions to the first moment $\Pi_{n=1}$ (top) and the second one (bottom). For comparison, we show HPQCD results (green triangles) $[11,16,19]$.

then take the average of the maximum and the minimum differences as our central value, with an uncertainty given by the half distance between the maximum and minimum. As read off at $L=6$ $\mathrm{fm}$ in Fig. 2, the correction is on the level of $2 \%(10 \%)$ for $\Pi_{1}\left(\Pi_{2}\right)$. We record these corrections for $\Pi_{n=1,2}$ in Table 2 . This correction increases rapidly with moment number, therefore we have chosen not to quote moments beyond the second one.
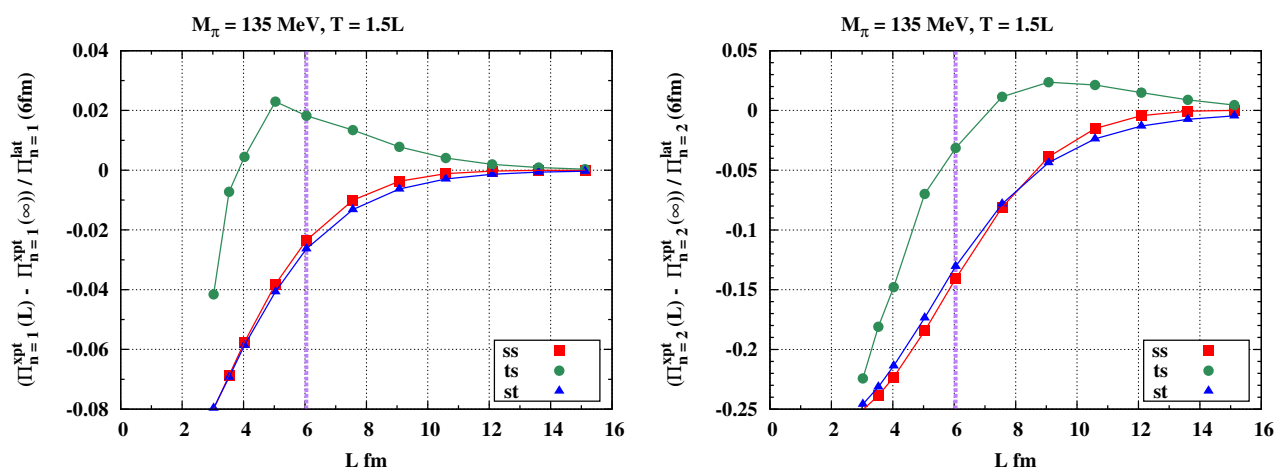

Figure 2: The FV of the first (left) and second (right) moments estimated by the $\chi \mathrm{PT}$.

Compared to phenomenological determinations [7,21], our $m_{d}=m_{u}$ calculation without QED is missing a number of effects associated with the isospin breaking: $\rho-\omega$ and $\rho-\gamma$ mixing, final state radiation, and the $\pi_{0} \gamma$ and $\eta \gamma$ contributions. This leads to a correction of $1.3 \%$ of the result for $a_{\mu}^{\mathrm{LO}-\mathrm{HVP}}$ (for details, see [1]). However, a competing effect enters. In our calculation without electromagnetism, the charged pion has a mass which is smaller than its physical value. As a result, the two-pion contribution to $a_{\mu}^{\mathrm{LO}-\mathrm{HVP}}$ is enhanced. This leads to a correction whose sign is opposite to the correction associated with the sum of effects discussed above. A phenomenological 


\begin{tabular}{lcc}
\hline \hline & $\Pi_{1}\left[\mathrm{GeV}^{-2}\right]$ & $\Pi_{2}\left[\mathrm{GeV}^{-4}\right]$ \\
\hline light & $0.1653(17)(16)$ & $-0.295(10)(7)$ \\
strange & $6.57(1)(3) \times 10^{-2}$ & $-5.33(1)(4) \times 10^{-2}$ \\
charm & $40.3(2)(6) \times 10^{-4}$ & $-2.66(3)(11) \times 10^{-4}$ \\
disconnected & $-1.5(2)(1) \times 10^{-2}$ & $4.4(1.0)(0.4) \times 10^{-2}$ \\
\hline$I=0$ & $0.0167(2)(2)$ & $-0.018(1)(1)$ \\
$I=1$ & $0.0827(8)(8)$ & $-0.147(5)(4)$ \\
total & $0.0993(10)(9)$ & $-0.165(6)(4)$ \\
\hline$I=1$ FV corr. & $0.0006(23)$ & $-0.016(10)$ \\
total + FV corr. & $0.0999(10)(9)(23)(13)$ & $-0.181(6)(4)(10)(2)$ \\
\hline \hline
\end{tabular}

Table 2: Summary table for $\Pi_{n=1,2}$ [1]. For the lattice results without FV corrections (first seven lines), the first and second brackets are the statistical and lattice systematic uncertainties, respectively. In the following two lines, we include an estimate of FV corrections obtained using LO $\chi \mathrm{PT}$ (see text). In the final results on the last line, the uncertainties due to FV and isospin-breaking corrections are given in the third and fourth brackets, respectively.

description based on $e^{+} e^{-}$data indicates that their magnitudes are very close [22]. Thus, we assume here that the total correction is $(0.0 \pm 1.3) \%$, where we have taken the error to be of the typical size of the corrections themselves. Because of the dominant role of $\Pi_{1}$ in determining $a_{\mu}^{\mathrm{LO}-\mathrm{HVP}}$, one expects a tantamount correction on that coefficient. Inferring the correction on $\Pi_{2}$ is less direct, but we assume here that it is of the same size as for $\Pi_{1}$. Thus, we add $(0.0 \pm 1.3) \%$ of $\Pi_{n=1,2}$ to our results for these quantities, after they have been corrected for finite-volume effects. Putting everything together we quote our final results for the first two moments in the last row of Table 2. Combining all four errors in quadrature, we obtain $\Pi_{1}\left(\Pi_{2}\right)$ with a total uncertainty of $2.9 \%(7.2 \%)$.

A phenomenological determination of $\Pi_{1}$ and $\Pi_{2}$ is has only become available recently [23]. Taking their "data direct" results, which are obtained from an interpolation of $e^{+} e^{-} \rightarrow$ hadrons data, and converting them to our conventions, we get $\Pi_{1}=0.0990(7) \mathrm{GeV}^{-2}$ and $\Pi_{2}=-0.2057(16)$ $\mathrm{GeV}^{-4}$. These numbers can be compared to our final results, i.e. those given in the last row of Table 2. In absolute value, their result for $\Pi_{1}$ is 0.3 combined standard deviations smaller than ours and for $\Pi_{2}, 1.9 \sigma$ larger. The latter might be due to an underestimate of FV corrections in our determination of the second moment, or some problem with the experimental data used in the phenomenological analysis of [23].

\section{Acknowledgments}

LL thanks M. Benayoun, C. Davies, F. Jegerlehner, C. Lehner, H. Leutwyler and E. de Rafael for very informative discussions. Computations were performed on JUQUEEN and JUROPA at Forschungszentrum Jülich, on Turing at the Institute for Development and Resources in Intensive Scientific Computing (IDRIS) in Orsay, on SuperMUC at Leibniz Supercomputing Centre in München, on Hermit at the High Performance Computing Center in Stuttgart. This project was supported by in part by the OCEVU Laboratoire d'excellence (ANR-11-LABX-0060) and the A*MIDEX Project (ANR-11-IDEX-0001-02), which are funded by the "Investissements d'Avenir" 
French government program and managed by the "Agence nationale de la recherche" (ANR), by the Gauss Centre for Supercomputing e.V and by the GENCI-IDRIS supercomputing grant No. 52275 .

\section{References}

[1] Sz. Borsanyi, Z. Fodor, T. Kawanai, S. Krieg, L. Lellouch, R. Malak, K. Miura, K.K. Szabo, C. Torrero, B. Toth, arXiv:1612.02364 [hep-lat].

[2] T. Kawanai et.al., [Budapest-Marseille-Wuppertal Collaboration], PoS LATTICE2016, 171 (2017).

[3] G. W. Bennett et al. [Muon g-2 Collaboration], Phys. Rev. D 73 (2006) 072003.

[4] F. Gray [New (g-2) Collaboration], J. Phys. Conf. Ser. 312 (2011) 102006; M. Otani (E34), JPS Conf. Proc. 8 (2015) 025008.

[5] T. Blum et al., arXiv:1311.2198 [hep-ph].

[6] J. Beringer et al. (Particle Data Group), Phys. Rev. D86 (2012) 010001.

[7] M. Davier, A. Hoecker, B. Malaescu and Z. Zhang, Eur. Phys. J. C 71 (2011) 1515 Erratum: [Eur. Phys. J. C 72 (2012) 1874]; S. Eidelman and F. Jegerlehner, Z. Phys. C 67 (1995) 585.

[8] T. Blum, Phys. Rev. Lett. 91 (2003) 052001.

[9] M. Luscher and P. Weisz, Commun. Math. Phys. 97 (1985) 59 Erratum: [Commun. Math. Phys. 98 (1985) 433].

[10] C. Morningstar and M. J. Peardon, Phys. Rev. D 69 (2004) 054501.

[11] C. T. H. Davies et al. [HPQCD Collaboration], Phys. Rev. Lett. 104 (2010) 132003.

[12] S. Aoki et al., arXiv:1607.00299 [hep-lat].

[13] M. Luscher, JHEP 1008 (2010) 071 Erratum: [JHEP 1403 (2014) 092].

[14] S. Borsanyi et al., JHEP 1209 (2012) 010.

[15] J. S. Bell and E. de Rafael, Nucl. Phys. B 11 (1969) 611; E. de Rafael, Phys. Lett. B 736 (2014) 522.

[16] B. Chakraborty et al. [HPQCD Collaboration], Phys. Rev. D 89 (2014) no.11, 114501.

[17] M. Luscher, Nucl. Phys. B 364 (1991) 237.

[18] S. Durr et al., Science 322 (2008) 1224; S. Borsanyi et al., Science 347 (2015) 1452.

[19] B. Chakraborty et al. [HPQCD Collaboration], arXiv:1601.03071 [hep-lat].

[20] C. Aubin, T. Blum, P. Chau, M. Golterman, S. Peris and C. Tu, Phys. Rev. D 93 (2016) no.5, 054508; A. Francis, B. Jaeger, H. B. Meyer and H. Wittig, Phys. Rev. D 88 (2013) 054502.

[21] K. Hagiwara, R. Liao, A. D. Martin, D. Nomura and T. Teubner, J. Phys. G 38 (2011) 085003; F. Jegerlehner, EPJ Web Conf. 118 (2016) 01016.

[22] M. Benayoun and F. Jegerlehner, private communication, November 2016.

[23] M. Benayoun, P. David, L. DelBuono and F. Jegerlehner, arXiv:1605.04474 [hep-ph]. 